\title{
L'accès aux soins de santé pour les personnes handicapées : plus que des lieux accessibles
}

$\mathrm{U}$ n Canadien sur huit - soit plus de 3,5 millions de personnes - vit avec une incapacité ${ }^{1}$. Grâce aux progrès réalisés en prestation des soins actifs et à cause du vieillissement de la population, le nombre de personnes handicapées au Canada continuera d'augmenter. On accorde toutefois peu d'attention aux besoins de cette importante population de patients.

Les discussions récentes sur les soins de santé au Canada ont porté principalement sur deux enjeux, soit l'utilisation des services privés et l'apparition possible d'un système de santé à deux vitesses, et les temps d'attente pour les services. Lorsqu'on s'intéresse à l'accessibilité des soins de santé pour les personnes handicapées, on constate toutefois que le Canada a déjà un système de santé à deux vitesses. Aussi important que soit l'accès aux soins en temps opportun, il est d'importance primordiale pour cette population de patients d'avoir accès aux soins médicalement nécessaires.

Les personnes handicapées peuvent parfois avoir besoin de soins complexes, mais beaucoup de leurs besoins fondamentaux sont les mêmes que ceux de la population en général. Or, les personnes handicapées ne reçoivent pas le même niveau de soins primaires et préventifs que les autres patients ${ }^{2}$. On ne leur fournit pas régulièrement des interventions de routine comme un test de Pap ou un examen de la prostate. Il y a encore plus troublant : les personnes handicapées sont quatre fois plus susceptibles que les personnes en bonne santé de signaler qu'elles n'arrivent pas à obtenir les soins médicaux nécessaires lorsqu'elles en ont besoin ${ }^{2}$.

Comme les incapacités reliées à la mobilité sont parmi les plus courantes au Canada, l'accès physique constitue un problème évident ${ }^{1}$. Mais même lorsqu'un édifice ou un bureau est accessible en fauteuil roulant, il suffit de regarder la table d'examen ordinaire qu'utilisent les médecins pour comprendre que la plupart de ces bureaux ne sont pas vraiment accessibles. Cela peut aider à expliquer pourquoi, souvent, des patients atteints d'incapacités physiques ne subissent pas les examens appropriés. De nouvelles mesures législatives comme la Loi de 2005 sur l'accessibilité pour les personnes handicapées de l'Ontario tiennent compte de l'accessibilité dans les hôpitaux, mais non dans les bureaux de médecins. L'absence de législation n'empêche néanmoins pas les médecins de suivre les lignes directrices disponibles pour rendre leurs bureaux accessibles pour leurs patients.

Or, l'élimination des seuls obstacles environnementaux ne suffit pas pour garantir l'accessibilité des soins de santé. La formation en médecine met beaucoup plus l'accent sur la guérison et la prévention des maladies que sur la prise en charge des patients atteints de maladies chroniques. Une vie sauvée réjouit beaucoup de gens, bien entendu, mais on célèbre rarement la prestation de soins de longue durée. Étudiants et résidents terminent leur formation après avoir consacré peu de temps à la prestation des soins chroniques et beaucoup ne savent pas trop comment interagir avec des patients handicapés.

Compte tenu des pressions qui s'exercent actuellement pour raccourcir les séjours en établissements de soins actifs, l'accès en temps opportun aux services de réadaptation ne devrait pas poser de problème. Or, l'accès aux programmes de réadaptation est souvent inexistant, même si les statistiques démontrent que ces retards réduisent considérablement le rétablissement fonctionnel des patients ${ }^{3}$. Les listes d'attente pour certaines interventions ont reçu une attention correspondant à l'intérêt des programmes politiques aussi bien qu'à l'existence de données cliniques solides. Personne ne peut s'élever contre les avantages de la disponibilité en temps opportun d'une arthroplastie pour les patients qui ont une arthrose dégénérative des articulations, ou d'activateurs tissulaires du plasminogène (AtP) pour les patients qui ont subi un accident vasculaire cérébral aigu. Cependant, comme les effectifs médicaux sont limités, l'utilisation accrue des services pour certaines interventions peut modifier l'éventail de services fournis et allonger par inadvertance les listes d'attente pour les patients qui ont autant de problèmes avec des incapacités chroniques. Même si on n'a pas suivi de près les temps d'attente pour les autres interventions, notre expérience indique que les périodes d'attente pour des évaluations orthopédiques ne figurant pas sur la liste des priorités se sont allongées depuis que les arthroplasties sont devenus prioritaires. De même, les ressources consacrées à la réadaptation après un accident vasculaire cérébral n'atteignent pas le niveau de celles rendues disponibles pour administrer des AtP.

Les patients handicapés ont souvent des problèmes médicaux complexes qui exigent d'aborder de multiples questions au cours d'une consultation avec un médecin. Ils et elles peuvent aussi avoir besoin de plus de temps et d'aide pour se déplacer et pour communiquer. Certaines provinces ont un code d'honoraires pour les soins chroniques qui rémunère en partie les médecins pour le temps de plus qu'ils doivent consacrer à ces patients. Toutefois, jusqu'à ce qu'il y ait suffisamment de médecins disponibles et que les médecins rémunérés à l'acte se jugent rémunérés suffisamment, il est possible que les patients handicapés continuent d'avoir de la difficulté à obtenir tous les soins dont ils et elles ont besoin.

Il est donc clair que les personnes handicapées - dont le nombre s'accroît - ont besoin d'un meilleur accès à des soins de santé de qualité. Outre les interventions à l'échelle du système, nous pouvons tous et toutes améliorer les soins que nous dispensons. Ensemble, nous pouvons faire une différence pour l'état de santé de cet important groupe de patients.

\section{Meridith B. Marks}

Médecine physique et réadaptation

Université d'Ottawa, Ottawa

Robert Teasell

Médecine physique et réadaptation

Université Western Ontario, London (Ont.)

Cet article a fait l'objet d'un examen par les pairs.

\section{RÉFÉRENCES}

I. Bureau de la condition des personnes handicapées, Développement social Canada. Vers l'intégration des personnes handicapées : un rapport du gouvernement $d u$ Canada. Ottawa : gouvernement du Canada; 2004. Disponible : www.sdcgc.ca/fr /pip/bcph/documents/versIntegrationo4/vipho4.pdf (consulté le ro juillet 2006).

2. Conseil canadien de développement social. Fiche d'information sur l'incapacité du CCDS : $\mathrm{N}^{\circ}$ 9, 2003. La santé et le bien-être des personnes handicapées. Disponible : www.ccsd.ca/drip/francais/recherche/disg/index.htm (consulté le ro juillet 2006).

3. Salter K, Jutai J, Hartley M, et al. Impact of early vs delayed admission to rehabilitation on functional outcomes in persons with stroke. J Rehabil Med 2006;38:113-7. 